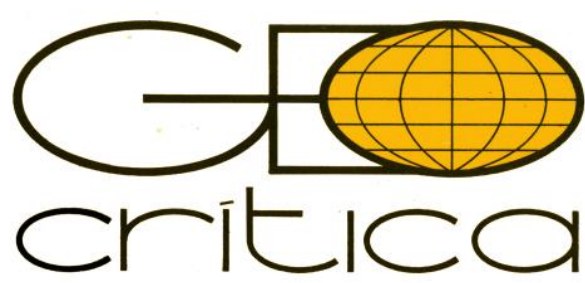

\title{
UNA CARTOGRAFÍA DE LOS FONDOS DOCUMENTALES DEL INGENIERO EDUARDO TORROJA A TRAVÉS DE SU TRAYECTORIA CIENTÍFICA, ACADÉMICA Y PROFESIONAL
}

\author{
Ramon Graus Rovira \\ Universitat Politècnica de Catalunya \\ ramon.graus@upc.edu
}

\begin{abstract}
Una cartografía de los fondos documentales del ingeniero Eduardo Torroja a través de su trayectoria científica, académica y profesional (Resumen)
\end{abstract}

La obra del ingeniero Eduardo Torroja sigue estimulando una copiosa producción de libros y artículos de revista. Asimismo, durante estos últimos veinte años sus fondos documentales se han ido ordenando $\mathrm{y}$, en parte, digitalizando. Algunos de estos fondos son muy utilizados, por inevitables y evidentes; sin embargo, existen fondos más dispersos que se convierten en esenciales si se atiene a su trayectoria científica, académica y profesional. Se trata de una cartografía no exhaustiva, pero suficientemente amplia y abierta para mostrar cómo se han documentado los últimos estudios y tiene la esperanza de animar a utilizar material poco estudiado de estos fondos en las nuevas investigaciones.

Palabras clave: arquitectura; Eduardo Torroja; estructuras laminares; hormigón armado; ingeniería.

Mapping of the document collections of engineer Eduardo Torroja over his scientific, academic and professional career (Abstract)

Engineer Eduardo Torroja's work continues to stimulate a substantial output of books and journal articles. Over the last twenty years, his document collections have been gradually organised and partly digitalised. Some are widely used because they are unavoidable and evident. However, other more dispersed collections exist that are crucial to a consideration of Torroja's scientific, academic and professional career. This is not an exhaustive map, but sufficiently broad and open to show how the latest research has been documented. It is hoped that it will encourage new research using the unpublished material that can be found in the collections.

Keywords: architecture; Eduardo Torroja; thin shell concrete structures; reinforced concrete; engineering. 


\section{Introducción}

El ingeniero de caminos español Eduardo Torroja Miret (1899-1961), desde el momento de su licenciatura en 1923 ha tenido un papel destacado en la arquitectura y la ingeniería del siglo XX. Y la clave de su éxito continuado ha sido precisamente su habilidad en ejercer su profesión, siempre al filo entre el arte y la técnica. En una mirada retrospectiva, el ingeniero Mario Salvadori reconocía "el papel que Torroja desempeñó durante los últimos treinta años tendiendo un puente en la brecha que siempre ha existido entre ingeniería civil y arquitectura" ${ }^{1}$.

Para trazar una sucinta cartografía de los fondos documentales que permitan estudiar biográfica e históricamente su figura es necesario reseguir su larga trayectoria científica y profesional, para lo que siguen siendo fundamentales las aproximaciones biográficas clásicas ${ }^{2}$. Este es el método que se utilizará en el artículo, con la confianza de que facilite una aproximación más rica a su legado.

\section{3-1927}

Eduardo Torroja inició su carrera trabajando para la Hidrocivil de José Eugenio Ribera, que había sido su profesor de Puentes en la Escuela de Ingenieros de Caminos, Canales y Puertos de Madrid. Ribera había sido uno de los introductores de la técnica del hormigón armado en España, tenía buenas conexiones en el extranjero y había creado una de las primeras empresas constructoras modernas. Rápidamente, Ribera reconoció el talento de Torroja y le reconoció la autoría de sus primeras obras dentro de la empresa, como los cajones de cimentación de los puentes de Sancti-Petri en Cádiz (1926) y de San Telmo en Sevilla (1926), o el acueducto del Tempul sobre el río Guadalete (1927). El fondo de Hidrocivil se puede dar por perdido y el catálogo de la exposición que le dedicó el Colegio de Ingenieros de Caminos, Canales y Puertos en 1982 sigue siendo la mejor introducción a Ribera ${ }^{3}$. En él se recoge material gráfico disperso y se publica una lista de sus publicaciones, la mayor parte de ellas en Revista de Obras Públicas, digitalizada hace ya unos años ${ }^{4}$. Ribera también publicaba en francés en Le Génie Civil, también digitalizada en Gallica ${ }^{5}$. Dentro de los artículos de Ribera de este período y en alguno de sus libros se pueden encontrar referencias o textos cortos de Torroja.

\section{8-1936}

En 1927, Eduardo Torroja abandonó su trabajo en Hidrocivil para establecer su propia Oficina Técnica de Proyectos y Dirección de Obras en Madrid, que mantuvo durante toda su vida. Su hijo, el también ingeniero de caminos y continuador de la Oficina Técnica, José Antonio Torroja Cavanillas, depositó en 2002, en régimen de comodato, los proyectos generados en la Oficina entre 1927 y 1965 en el Centro de Estudios Históricos de Obras Públicas y Urbanismo (CEHOPU), para crear el Fondo de Eduardo Torroja Miret. Se trata, pues, de la fuente

\footnotetext{
${ }^{1}$ Salvadori 1968, p. [57].

2 Arredondo et al. 1977, Tarragó 1979, Fernández Ordóñez y Navarro Vera 1999.

3 Tarragó 1982.

${ }^{4}$ Revista de Obras Públicas <http://ropdigital.ciccp.es/>.

${ }^{5}$ Gallica <https://gallica.bnf.fr/ark:/12148/cb34348662d/date〉.
} 
Una cartografía de los fondos documentales del ingeniero Eduardo Toroja...

documental más valiosa (conserva 609 unidades documentales) para conocer la gestación de cada una de las obras en las que intervino Torroja ${ }^{6}$.

Entre los investigadores que han estudiado el material de este archivo cabe destacar a Joaquín Antuña ${ }^{7}$ que dedicó su tesis doctoral a analizar específicamente los proyectos de edificación de la Oficina Técnica, y a la que le sucedieron diversos artículos divulgativos coincidiendo en el momento de ingreso del archivo al CEHOPU ${ }^{8}$.

Casi inmediatamente después de abrir la Oficina Técnica, en 1928, Torroja fue llamado a formar parte del equipo que el arquitecto Modesto López-Otero estaba seleccionando para el Gabinete Técnico de la Junta de Obras de la Ciudad Universitaria. López-Otero había reunido un heterogéneo grupo de jóvenes arquitectos de talento entre los que estaban Luis Lacasa, Manuel Sánchez Arcas, Miguel de los Santos o Agustín Aguirre. Torroja participó con todos ellos en las obras de infraestructura, en las obras de los edificios de las facultades y, después de la Guerra Civil, en la reconstrucción subsiguiente del campus. Pilar Chías estudió en su tesis doctoral la gestación de este gran campus universitario ${ }^{9}$. Parte del material se conserva en el Archivo del CEHOPU, pero también puede encontrarse documentación, las actas de las sesiones de la Junta Constructora, proyectos y presupuestos y fotografías en el Archivo General de la Universidad Complutense de Madrid ${ }^{10}$. Este material fue consultado por el equipo que preparó el último libro aparecido sobre la Ciudad Universitaria, y que publicó nuevo material gráfico ${ }^{11}$.

Durante estos años, Torroja diseñó y construyó las tres obras más influyentes de su carrera profesional: el mercado de Algeciras (1932-35) con Manuel Sánchez Arcas, el Hipódromo de La Zarzuela (1934-1936, 1941) con Carlos Arniches y Martín Domínguez, y el Frontón Recoletos (1935-36) con Secundino Zuazo. De estos arquitectos solo se mantiene con integridad el Archivo Zuazo, conservado en la Biblioteca Nacional de España ${ }^{12}$. El carácter innovador de las bóvedas laminares de hormigón armado que propuso para estas obras llevó a Eduardo Torroja a ensayar modelos reducidos para valorar empíricamente su comportamiento estructural. Con este objetivo fundó en 1934 Investigaciones de la Construcción, S.A. (ICON), una empresa que se especializó en la fabricación de instrumentos de precisión para laboratorios de materiales de construcción y en la instrumentación de modelos reducidos.

Durante estos años Eduardo Torroja empezó a registrar patentes, algunas sobre instrumental de ICON y otras más específicas sobre estructuras concretas. Pueden consultarse en el buscador de propiedad industrial de la Oficina Española de Patentes y Marcas ${ }^{13}$.

Finalmente, en 1934, fundó dos plataformas que le permitieron iniciar su larga trayectoria científica. De una conversación con José María Aguirre de Agromán, durante una visita de obras a la Ciudad Universitaria, donde este último era contratista, surgió la idea de crear el Instituto Técnico de la Construcción y Edificación (ITCE), cuyo comité ejecutivo fue formado por Modesto López-Otero, presidente, Alfonso Peña Bœuf, vicepresidente, Manuel Sánchez Arcas, José María Aguirre, Gaspar Blein y José Ángel Petrirena, vocales, y Eduardo Torroja,

\footnotetext{
${ }^{6}$ Fondo de Eduardo Torroja Miret <http://www.cehopu.cedex.es/etm/etm_index.htm>.

${ }^{7}$ Antuña $2003<$ http://oa.upm.es/1348/>.

${ }^{8}$ Antuña y Pedregal 2002, García García y Antuña 2006.

${ }^{9}$ Chías $1983<$ <http://oa.upm.es/10629/>.

${ }^{10}$ Junta Constructora de la Ciudad Universitaria <https://www.ucm.es/pagina-principal-archivo $>$.

${ }^{11}$ Rodríguez-López y Muñoz Hernández 2018.

${ }^{12}$ Archivo Zuazo <http://catalogo.bne.es/>.

${ }^{13}$ Oficina Española de Patentes y Marcas <http://historico.oepm.es/buscador.php>.
} 
secretario. El Instituto aspiraba a mejorar el sector de la construcción del país con cursos de especialización, investigación científica y el impulso de normas técnicas y estándares de los que el país carecía. La segunda plataforma fue complementaria a la primera, con el ingeniero Enrique García-Reyes creó la revista Hormigón y Acero, que terminaría convirtiéndose en el órgano oficial del ITCE. La colección completa de la revista se conserva digitalizada en la Biblioteca Digital Hispánica ${ }^{14}$.

La obra construida de Eduardo Torroja de este periodo fue fotografiada por encargo del mismo ingeniero por la fotógrafa alemana Sibylle von Kaskel (1905-2005) $)^{15}$. Su fotografía, muy próxima a la Nueva Visión, muestra la obra de Torroja desde la óptica de las vanguardias del momento. Si bien no se conserva su archivo fotográfico, sus fotografías fueron publicadas en la primera monografía que se autoeditó Torroja en $1936^{16}$.

\section{6-1939}

La llegada de la Guerra Civil paralizó las obras del Hipódromo de la Zarzuela, y durante la batalla de Madrid fueron dañados el Hipódromo y el Frontón Recoletos. La cubierta del Frontón no se pudo salvar. Torroja se desplazó de Madrid a Hondarribia, en zona sublevada, mientras recibía el encargo de Alfonso Peña Bœuf, ministro de Obras Públicas de Franco, de preparar, en plena guerra, una Instrucción de proyectos y obras de hormigón. En el año 1938, Torroja con otros socios fundó Obras Metálicas Electro-Soldadas, S. A. (OMES). Como indica su nombre, era una empresa especializada en la construcción con acero con uniones electrosoldadas, una absoluta novedad técnica en la época. Se trataba de una iniciativa que ya estaba pensando en la reconstrucción de puentes y otras infraestructuras cuando terminara la guerra. Tanto de ICON como de OMES se pueden localizar los datos que debían registrarse legalmente como entidades mercantiles ${ }^{17}$.

\section{|939-1945}

En julio de 1939 fue nombrado profesor en la Escuela de Caminos de Madrid de las asignaturas de "Cálculo de Estructuras" y de "Hormigón Armado", a partir de 1945 de "Teoría de Elasticidad" y "Resistencia de Materiales" y, unos años después, en 1953, de "Tipología Estructural" 18 . Pero aún más trascendental fue su nombramiento como director del Laboratorio Central de Ensayo de Materiales de Construcción (LCEMC) de la misma Escuela en noviembre de $1940^{19}$. Entre 1941 y 1947 Torroja diseñó y construyó un nuevo edificio para el Laboratorio; se trataba del espacio soñado por el Torroja de los años treinta, un lugar desde donde impulsar la investigación sobre múltiples aspectos de la construcción y, cómo no, poder continuar sus ensayos con modelos reducidos. Saénz Ridruejo se apoyó en los Anuarios de la Escuela, en las actas de la Junta de profesores y en los archivos del Ministerio de Fomento y del Colegio de Ingenieros de Caminos, Canales y Puertos ${ }^{20}$ para su investigación sobre la historia de la Escuela de Caminos de Madrid. Parte de la información del Laboratorio se debería encontrar en el

\footnotetext{
${ }^{14}$ Biblioteca Digital Hispánica <http://hemerotecadigital.bne.es/details.vm?q=id:0005269420\&lang=es >

15 Naranjo 1997, p. 28-31, 58-62, Fernández 2004, p. 60-71, Martínez 2000.

16 Torroja 1936.

${ }^{17}$ Registro Mercantil de Madrid. ICON, Hoja 6850, $1^{\text {a }}$ inscripción, 24 de abril de 1934 y OMES, hoja 244, $1^{\text {a }}$ inscripción, 14 de febrero de 1944 <https://www.rmercantilmadrid.com/RMM/Tramites/Notas_Simples.aspx >.

${ }^{18}$ Sáenz Ridruejo 2016, p. 429, 536.

${ }^{19}$ Sáenz Ridruejo 2016, p. 456-457.

${ }^{20}$ Sáenz Ridruejo 2016, p. 18.
} 
Una cartografía de los fondos documentales del ingeniero Eduardo Toroja...

CEDEX, ya que a partir de 1957 pasó a formar parte de este organismo, con el nombre actual de Laboratorio Central de Estructuras y Materiales ${ }^{21}$.

Durante estos años, con el concurso de OMES, Torroja reconstruye diversos puentes, entre los que cabe destacar la finalización del gran viaducto Martín Gil sobre el Esla, en la provincia de Zamora. La obra y la inauguración del puente fue filmada por el gran documentalista Fernando López Heptener, que trabajaba tanto para Iberduero como para NO-DO ${ }^{22}$.

Asimismo, con la complicidad del ministro Peña Bœuf, que había sido uno de los fundadores del ITCE, Torroja consiguió reflotar el Instituto creado en 1934 dentro de las nuevas estructuras científicas de la dictadura. En junio de 1940 asociaron el ITCE como centro adherido del Patronato Juan de la Cierva del CSIC y en 1945 se incorporó definitivamente en él.

\section{| 946- | 96 |}

Torroja había aceptado la absorción por el Estado del ITCE en 1945 con la confianza de encontrar vías públicas y privadas de financiación, en ese momento de traspaso el Instituto pasó a denominarse Instituto Técnico de la Construcción (ITC). Estos fondos económicos llegaron cuando se decidió fusionar su ITC con el Instituto del Cemento ${ }^{23}$, un instituto que recibía financiación de las empresas cementeras a través de un canon por tonelada de cemento producida. Así se creaba en 1949 el Instituto Técnico de la Construcción y del Cemento (ITCC). Lino Camprubí ha estudiado en profundidad las redes de contactos que permitieron su creación ${ }^{24}$, entre las fuentes que ha consultado cabe destacar una parte del material conservado en el Archivo General de la Administración ${ }^{25}$. Torroja centró sus esfuerzos en hacer realidad la nueva sede del ITCC en el Pinar de Chamartín de Madrid, con unos laboratorios excepcionales para la época, con la confianza en mejorar el sector de la construcción del país. La compra del nuevo solar se realizó en 1950, las obras se iniciaron en 1951, el inicio del traslado se produjo en 1953 y su inauguración definitiva con la presencia de Franco se produjo en $1958^{26}$. Una de las fuentes documentales de este período productivo de Torroja es el mismo archivo de dirección del ITCC, ahora llamado Instituto de Ciencias de la Construcción Eduardo Torroja. A partir de 2007 se llevó a cabo el proyecto de investigación El fondo documental generado por Eduardo Torroja en el IETcc como memoria histórica en el proceso de transferencia tecnológica en Ciencias de la Construcción con el objetivo de ordenar el material del fondo relacionado con su primer director. La investigación permitió crear una interesante base de datos, consultable en línea en formato pdf $^{27}$, y afloró material inédito sobre la construcción de la sede del ITCC, que fue analizado y publicado detalladamente ${ }^{28}$.

\footnotetext{
${ }^{21}$ Astudillo 1998, p. 52.

${ }^{22}$ Cebrián 1994 <http://bibliotecadigital.jcyl.es/es/catalogo_imagenes/grupo.cmd?path=10162310>.

${ }^{23}$ Asensio et al. 2012, p. 11-13.

${ }^{24}$ Camprubí 2017, p. 71-92.

${ }^{25}$ Pares <http://pares.culturaydeporte.gob.es/inicio.html>.

${ }^{26}$ Cassinello 2012.

${ }^{27}$ HUM2007-65543/HIST: El fondo documental... $2010<$ https://www.ietcc.csic.es/informaciongeneral/historia/archivo-historico/>.

${ }^{28}$ Azorín 2012, Azorín, Cassinello y Monjo 2012

<http://informesdelaconstruccion.revistas.csic.es/index.php/informesdelaconstruccion/article/view/1711/1919>, Azorín y Monjo 2012, Cassinello 2012.
} 
En 1948 Torroja había creado aún en el seno del ITC la revista Informes de la Construcción, una especie de reedición de Hormigón y Acero, y que a partir del número 12, de julio de 1949, ya se publicaría como órgano del recientemente creado ITCC $^{29}$. Esta fue la plataforma en lengua española que estará a disposición de Torroja para divulgar la ciencia y la tecnología de la construcción, así como para difundir su particular interés por la arquitectura moderna internacional $^{30}$. La revista es una fuente inagotable de datos ${ }^{31}$, especialmente sus secciones de Noticias del Instituto y de Noticias Internacionales donde se pueden reseguir los viajes innumerables que emprende Torroja por Europa y América, una vez finalizada la Segunda Guerra Mundial. La revista está solamente digitalizada a partir del número 87 de $1957^{32}$, pero se han publicado los índices completos ${ }^{33}$.

La confianza que depositaba el régimen militar franquista en los ingenieros elevó a Torroja a la presidencia de la Empresa Nacional Hidroeléctrica del Ribagorzana (ENHER) en 1946. Le acompañaba como director ejecutivo el también ingeniero Victoriano Muñoz Oms, que conocía muy bien la cuenca hidrográfica de la Noguera Ribagorçana, y que ENHER deseaba explotar para la producción de electricidad. Los dos ingenieros planificaron la transformación y sistematización del río y Torroja se reservó el diseño de la presa de Canelles. Lino Camprubí también ha estudiado el papel de Torroja en esta aventura ${ }^{34}$. El fondo documental de ENHER se conserva en la Fundación ENDESA ${ }^{35}$.

A través del LCEMC y del ITCC Torroja estableció una cantidad innumerable de contactos con asociaciones profesionales ${ }^{36}$. En cada una de estas asociaciones se encontraría información de su presencia y seguramente correspondencia. Cabe destacar su destacada participación, a partir del final de la Segunda Guerra Mundial, en RILEM ${ }^{37}$, IABSE$^{38}$, CEB, FIP $^{39}$ e IASS ${ }^{40}$. Esta última merece una explicación más detenida porque nació de su interés particular en las cubiertas laminares de hormigón armado, fue creada durante un simposio técnico en Madrid en septiembre de 1959 y convirtió el LCEMC en la sede de su secretariado ${ }^{41}$. En la actualidad, el secretariado de la IASS sigue siendo llevado por el Laboratorio Central de Estructuras y Materiales del CEDEX en Madrid.

Como se ha argumentado recientemente ${ }^{42}$, Torroja fue reconocido en el ámbito de la arquitectura moderna gracias a sus contactos con arquitectos norteamericanos como Frank Lloyd Wright y Richard Neutra. En este reconocimiento jugaron un papel muy destacado las

\footnotetext{
${ }^{29}$ Oteiza, Azorín y Salas 2008 <https://doi.org/10.3989/ic.2008.v60.i510.736>, Azorín y Monjo 2012, p. 44-45.

${ }^{30}$ Esteban Maluenda $2008<$ https://doi.org/10.3989/ic.2008.v60.i510.733>.

31 Cassinello $2008<$ https://editorial.csic.es/publicaciones/libros/11574/978-84-00-08654-1/el-espiritu-impresode-una-idea-exposicion-conmemo.html $>$.

32 Informes de la Construcción <https://doi.org/10.3989/ic.14.113>.

${ }^{33}$ Ponce Ortiz de Insagurbe y Sánchez Sánchez 2006 <https://editorial.csic.es/publicaciones/libros/11339/978-8400-08503-2/construir-el-siglo-Xx-con-informes-de-la-construcc.html $>$.

${ }^{34}$ Camprubí 2017, p. 111-130.

${ }^{35}$ Fondo Histórico ENDESA <https://www.fundacionendesa.org/es/cultura/a201703-fondo-historicopatrimonio>.

36 Thürlimann 2000.

37 RILEM (Réunion Internationale des Laboratoires d'Essais et des Recherches sur les Matériaux et les Constructions) <https://www.rilem.net/>.

${ }^{38}$ IABSE (International Association for Bridge and Structural Engineering) <https://iabse.org/>.

${ }^{39}$ CEB (Comité Européen du Béton) y FIP (Fédération Internationale de la Précontrainte) se unieron en 1996 para formar la FIB (Fédération Internationale du Béton).

${ }^{40}$ IASS (International Association for Shell and Spatial Structures) <https://doi.org/10.3989/ic.14.113>.

${ }^{41}$ Astudillo 2014 , Isler 2003.

${ }^{42}$ Graus y Navas-Ferrer 2019 <http://hdl.handle.net/2117/175584>.
} 
Una cartografía de los fondos documentales del ingeniero Eduardo Toroja...

instantáneas del Hipódromo de la Zarzuela que realizó el fotógrafo norteamericano G. E. Kidder Smith ${ }^{43}$, ya que fueron publicadas en el gran altavoz de la arquitectura moderna estadounidense que era Architectural Forum ${ }^{44}$. Martín-Sáiz ${ }^{45}$ ha mostrado como el ingeniero checo Jaroslav J. Polívka ${ }^{46}$ (colaborador de Wright desde la Johnson Wax Research Tower hasta el Guggenheim Museum), que conocía a Torroja, lo presentó a Wright en abril de 1950. En la University at Buffalo ${ }^{47}$ se conservan nueve fotografías de la primera visita de Torroja y unas memorias de Polívka en las que detalla su relación con el ingeniero español ${ }^{48}$. Asimismo, Larripa y Larumbe ${ }^{49}$ encontraron y publicaron la transcripción de la participación de Torroja en un "Talk to the Taliesin Fellowship" en octubre de 1954, que ahora se conserva en la Avery Library $^{50}$. Torroja volvió a visitar los Estados Unidos de América en 1958 y 1960 y, por tanto, queda mucho trabajo por hacer. Precisamente, en 1958, la monografía The structures of Eduardo Torroja ${ }^{51}$ fue publicada por el grupo editor F.W. Dodge que era el propietario de la revista Architectural Record, mientras The University of California publicaba Philosophy of structures $^{52}$, la traducción al inglés de Razón y ser de los tipos estructurales ${ }^{53}$, publicada un año antes en Madrid. Los borradores del libro se conservan en el Archivo Eduardo Torroja Miret del CEHOPU, con la referencia 807. A está traducción le siguieron la japonesa (1960) y la alemana (1961). Eduardo Torroja falleció en junio de 1961 y ya no vio en vida las traducciones italiana (1966) y francesa (1969).

\section{Consideraciones finales}

No era el objetivo del texto comentar su obra escrita. La lista más completa de textos sigue siendo la que se publicó en 1977 por sus colaboradores más próximos ${ }^{54}$. Cabe destacar que el proyecto de investigación "La arquitectura española en los medios de comunicación internacionales: publicaciones, exposiciones, congresos (primera parte: 1940-1975)", AEMCI 1 (HAR2017-85205-P) ha preparado una base de datos abierta en formato Mendeley ${ }^{55}$ sobre aquellas revistas y libros extranjeros que publicaron arquitectura española entre 1940 y 1975. Entre estas publicaciones se pueden encontrar más de un centenar referidas a Torroja.

Por otro lado, en el año 2004 se creó la Fundación Eduardo Torroja ${ }^{56}$, que entre otras actividades mantiene el Museo Eduardo Torroja en el Hipódromo de la Zarzuela de Madrid, en la que se pueden contemplar maquetas de sus obras principales. Asimismo, en el Museo Virtual del CEDEX puede consultarse información y fotografías de las mismas maquetas, que habían sido

\footnotetext{
${ }^{43}$ Maggi 2016.

${ }^{44}$ Smith 1950.

${ }^{45}$ Martín-Sáiz $2012<$ http://hdl.handle.net/10803/78010> y 2013

$<$ http://informesdelaconstruccion.revistas.csic.es/index.php/informesdelaconstruccion/article/view/2815/3123>.

${ }^{46}$ Zikmund-Lender 2018.

${ }^{47}$ Polívka Papers <https://findingaids.lib.buffalo.edu/repositories/2/resources/704>.

${ }^{48}$ Polívka 1957 <https://findingaids.lib.buffalo.edu/repositories/2/archival_objects/225469>

${ }^{49}$ Larripa y Larumbe 2016.

${ }^{50}$ The Frank Lloyd Wright Foundation Archives <https://clio.columbia.edu/catalog/12471384>.

51 Torroja $1958 b$.

52 Torroja 1958a.

53 Torroja 1957.

54 Arredondo et al. 1977, 336-363.

55 Spanish architecture seen worldwide <https://www.mendeley.com/community/spanish-architecture-seenworldwide/>. [Es recomendable abrirse una cuenta Mendeley y descargarse el gestor de referencias para tener acceso a todas las funcionalidades].

${ }^{56}$ Fundación Eduardo Torroja <http://www.fundacioneduardotorroja.org/index.php/es/>.
} 
preparadas ex profeso para la Exposición Torroja que comisarió José Antonio Torroja Cavanillas $^{57}$.

En definitiva, su actividad científica, pedagógica y profesional es inmensa y se despliega en múltiples facetas. Sin embargo, para ahondar en el estudio histórico de Eduardo Torroja y su obra sería necesario dar un paso más y acceder con detalle y profundidad a su correspondencia; una parte la conserva el CEHOPU, otra parte la familia, y mucha más está desperdigada por las instituciones y contactos personales que hemos esbozado en estas líneas a la espera de nuevas investigaciones.

\section{Agradecimientos}

La investigación ha sido posible gracias al apoyo del Ministerio de Ciencia e Investigación, del Ministerio de Universidades y el Fondo Europeo de Desarrollo Regional de la Unión Europea (FEDER), en el marco del proyecto "La arquitectura española en los medios de comunicación internacionales: publicaciones, exposiciones, congresos (primera parte: 1940-1975)", AEMCI 1

(HAR2017-85205-P).

<http://www.spanisharchitecturenetwork.upc.edu/>.

\section{Bibliografía}

ANTUÑA, Joaquín. Las estructuras de edificación de Eduardo Torroja Miret [en línea]. Madrid: Universidad Politécnica de Madrid, 2003. Tesis doctoral dirigida por Ricardo Aroca. <http://oa.upm.es/1348/>. [Consulta: 1 septiembre 2020].

ANTUÑA, Joaquín y PEDREGAL, José Manuel. El Archivo de don Eduardo Torroja. En: Ingeniería Civil. 2002, no. 127, p. 3-9.

ARCHIVO ZUAZO (DIBZ/58), Catálogo [en línea] Servicio de Dibujos y Grabados de la Biblioteca Nacional de España. <http://catalogo.bne.es/>. [Consulta: 1 septiembre 2020].

ARREDONDO, Francisco et al. La obra de Eduardo Torroja. Madrid: Instituto de España. Colección Cultura y Ciencia, 1977.

ASENSIO, Eloy et al. Los inicios de la investigación en la química del cemento en España. En: Cemento-Hormigón. 2012, no. 949, p. 8-31.

ASTUDILLO, Rafael. Centenario del Laboratorio Central de Estructuras y Materiales del CEDEX. En: Revista de Obras Públicas. 1998, vol. 145, no. 3378, p. 51-56.

ASTUDILLO, Rafael. Eduardo Torroja y la International Association for Shell and Spatial Structures (IASS). En: Informes de la Construcción. 2014, vol. 66, no. 536, p. e037. <DOI: https://doi.org/10.3989/ic.14.113>.

AZORÍN, Virtudes. Los inicios de la investigación en Ciencias de la Construcción en España: Una aproximación histórica a través del Archivo Histórico de la dirección del Instituto de Ciencias de la Construcción Eduardo Torroja. En: V. AZORÍN, P. CASSINELLO y J. MONJO,

\footnotetext{
${ }^{57}$ Torroja Cavanillas 1999 <http://www.museovirtual.cedex.es/detalle-exposicion-cedex.html?id=10>.
} 
eds., Archivo Eduardo Torroja: de la investigación en construcción y de su innovador hábitat (ITCC). Madrid: Instituto de Ciencias de la Construcción Eduardo Torroja, Consejo Superior de Investigaciones Científicas, 2012, p. 15-23.

AZORÍN, Virtudes, CASSINELLO, Pepa y MONJO, Juan. Archivo Eduardo Torroja: La sede del itcc (1949-1953): Inéditos anteproyectos previos a su construcción. En: Informes de la Construcción. 2012, vol. 64, no. 525, p. 5-18. <DOI: https://doi.org/10.3989/ic.11.023>.

AZORÍN, Virtudes y MONJO, Juan. Cuarenta años de investigación en Ciencias de la Construcción en España. En: V. AZORÍN, P. CASSINELLO y J. MONJO, eds., Archivo Eduardo Torroja: de la investigación en construcción y de su innovador hábitat (ITCC). Madrid: Instituto de Ciencias de la Construcción Eduardo Torroja, Consejo Superior de Investigaciones Científicas, 2012, p. 25-73.

BIBLIOTECA DIGITAL HISPÁNICA, Catálogo [en línea]: Hormigón y Acero. <http://hemerotecadigital.bne.es/details.vm?q=id:0005269420\&lang=es>. [Consulta: 1 septiembre 2020].

CAMPRUBÍ, Lino. Los ingenieros de Franco: Ciencia, catolicismo y Guerra Fría en el Estado franquista. Barcelona: Crítica, 2017. Contrastes.

CASSINELlo, Pepa, ed. El espíritu impreso de una idea: Catálogo de la Exposición conmemorativa 60 años de la revista "Informes de la Construcción»= The spirit of an idea in print: Catalogue of the commemorative Exhibition 60 years «Informes de la Construcción» [en línea]. Madrid: Asociación de Miembros del Instituto Eduardo Torroja, CSIC, 2008. $<$ https://editorial.csic.es/publicaciones/libros/11574/978-84-00-08654-1/el-espiritu-impresode-una-idea-exposicion-conmemo.html>. [Consulta: 1 septiembre 2020].

CASSINELLO, Pepa. Eduardo Torroja y el innovador hábitat de su modelo de investigación: La sede del ITCC (1949-1953): anteproyecto, proyecto y construcción. En: V. AZORÍN, P. CASSINELlO y J. MONJO, eds., Archivo Eduardo Torroja: de la investigación en construcción y de su innovador hábitat (ITCC). Madrid: Instituto de Ciencias de la Construcción Eduardo Torroja, Consejo Superior de Investigaciones Científicas, 2012, p. 75153.

CEBRIÁN, Mariano. Cine documental e informativo de empresa: 50 años de producción de Fernando López Heptener en Iberduero y NO-DO [en línea]. Madrid: Síntesis, 1994. $<$ http://bibliotecadigital.jcyl.es/es/catalogo_imagenes/grupo.cmd?path=10162310>.

[Consulta: 1 septiembre 2020].

CHÍAS, Pilar. La Ciudad Universitaria de Madrid: Planeamiento y realización [en línea]. Madrid: Universidad Politécnica de Madrid, 1983. Tesis doctoral dirigida por Antonio Bonet Correa. <http://oa.upm.es/10629/>. [Consulta: 1 septiembre 2020].

ESTEBAN MALUENDA, Ana. Informes de la Construcción: más que una revista técnica. En: Informes de la Construcción. 2008, vol. 60, no. 510, p. 87-102. <DOI: https://doi.org/10.3989/ic.2008.v60.i510.733>. 
FERNÁNDEZ, Horacio, ed. Variaciones en España: Fotografía y arte: 1900-1980. Madrid: La Fábrica, Fundación MARCO, CAAM, 2004.

FERNÁNDEZ ORDÓÑEZ, José Antonio y NAVARRO VERA, José Ramón. Eduardo Torroja, ingeniero $=$ Eduardo Torroja, engineer. Madrid: Pronaos, 1999.

FIB (Fédération Internationale du Béton), Web institucional [en línea]. <https://www.fibinternational.org/>. [Consulta: 1 septiembre 2020].

FONDO DE EDUARDO TORROJA MIRET, Inventario [en línea], ES.28079.CEHOPU/1.02. Madrid: CEDEX, <http://www.cehopu.cedex.es/etm/etm_index.htm>. [Consulta: 1 septiembre 2020].

FONDO HISTÓRICO ENDESA, Subfondo ENHER [en línea]. <https://www.fundacionendesa.org/es/cultura/a201703-fondo-historico-patrimonio>.

[Consulta: 1 septiembre 2020].

FRANK LLOYD WRIGHT FOUNDATION ARCHIVES, The (The Museum of Modern Art Avery Architectural \& Fine Arts Library, Columbia University, New York), Fellowship talk transcripts, ca. 1890-1960 [en línea]. <https://clio.columbia.edu/catalog/12471384>. [Consulta: 1 septiembre 2020].

FUNDACIÓN EDUARDO TORROJA, Web institucional [en línea]. <http://www.fundacioneduardotorroja.org/index.php/es/>. [Consulta: 1 septiembre 2020].

GALLICA, Bibliothèque numérique de la Bibliothèque nationale de France [en línea]: Le Génie civil. <https://gallica.bnf.fr/ark:/12148/cb34348662d/date>. [Consulta: 1 septiembre 2020].

GARCÍA GARCÍA, Isabel María y ANTUÑA, Joaquín. Las fuentes de la historia de la construcción. Los archivos de autor. El archivo Eduardo Torroja. En: Ingeniería Civil. 2006, no. 141 , p. 109-115.

GRAUS, Ramon y NAVAS-FERRER, Teresa. Eduardo Torroja: el camino hacia su reconocimiento internacional. En: Redes Internacionales de la Arquitectura Española: 1er seminario AEMCI [en línea]. Barcelona: Iniciativa Digital Politècnica, 2019, p. 10-11. <http://hdl.handle.net/2117/175584>. [Consulta: 1 septiembre 2020].

HUM2007-65543/HIST: El fondo documental generado por Eduardo Torroja en el IETcc como memoria histórica en el proceso de transferencia tecnológica en Ciencias de la Construcción [en línea]. Madrid: Instituto Eduardo Torroja de Ciencias de la Construcción, CSIC, 2010. <https://www.ietcc.csic.es/informacion-general/historia/archivo-historico/>. [Consulta: 1 septiembre 2020].

IABSE (International Association for Bridge and Structural Engineering), Web institucional [en línea]. <https://iabse.org/>. [Consulta: 1 septiembre 2020].

IASS (International Association for Shell and Spatial Structures), Web institucional [en línea]. <https://www.iass-structures.org/>. [Consulta: 1 septiembre 2020]. 
INFORMES DE LA CONSTRUCCIÓN, Repositorio de números publicados [en línea]. $<$ http://informesdelaconstruccion.revistas.csic.es>. [Consulta: 1 septiembre 2020].

ISLER, Heinz. Eduardo Torroja and the IASS, the International Association for Shell Structures. En: F. LEVI, M. A. CHIORINO y C. BERTOLINI CESTARI, eds., Eduardo Torroja: From the philosophy of structures to the art and science of building: International Seminar: Politecnico di Torino. Milano: FrancoAngeli, 2003, p. 48-53.

JUNTA CONSTRUCTORA DE LA CIUDAD UNIVERSITARIA, Archivo General de la Universidad Complutense de Madrid [en línea]. <https://www.ucm.es/pagina-principalarchivo>. [Consulta: 1 septiembre 2020].

LARRIPA, Víctor y LARUMBE, Teresa. Torroja y Wright: Una mirada común. En: J. M. POZO, ed., Arquitectura importada y exportada en España y Portugal (1925-1975): Actas del X Congreso Nacional de Arquitectura Moderna Española 2016. Pamplona: Universidad de Navarra, T6 Ediciones, 2016, p. 315-330.

MAGGI, Angelo. The visual transmission of European architecture by George Everard Kidder Smith. En: R. A. ALCOLEA y J. TÁRRAGO-MINGO, eds., Congreso internacional: Inter photo arch «Interpretaciones». Pamplona: Universidad de Navarra, 2016, p. 140-151.

MARTÍN-SÁIZ, Diego. El Guggenheim Museum de New York: Interpretación del papel de la estructura a través de la colaboración entre Frank Lloyd Wright y Jaroslav J. Polivka [en línea]. Barcelona: Universitat Politècnica de Catalunya, 2012. Tesis doctoral dirigida por Robert Brufau. <http://hdl.handle.net/10803/78010>. [Consulta: 1 septiembre 2020].

MARTÍN-SÁIZ, Diego. Jaroslav J. Polivka y el Guggenheim Museum de New York. En: Informes de la Construcción. 2013, vol. 65, no. 531, p. 261-274. <DOI: https://doi.org/10.3989/ic.12.036>.

MARTÍNEZ, Covadonga. La baronesa Sibylle von Kaskel: Un recorrido por la vanguardia fotográfica (1930-1950). Sevilla: Albores, 2020.

NARANJO, Joan, ed. Les avantguardes fotogràfiques a Espanya (1925-1945) = Avant-garde photography in Spain (1925-1945). Barcelona: Fundació «la Caixa», 1997.

OFICINA ESPAÑOLA DE PATENTES Y MARCAS, Web Histórico [en línea]. <http://historico.oepm.es/buscador.php>. [Consulta: 1 septiembre 2020].

OTEIZA, Ignacio, AZORÍN, Virtudes y SALAS, Julián. «Informes de la Construcción»: pasado, presente y futuro. En: Informes de la Construcción. 2008, vol. 60, no. 510, p. 119-129. <DOI: https://doi.org/10.3989/ic.2008.v60.i510.736>.

PARES, Base de datos, <http://pares.culturaydeporte.gob.es/inicio.html>. [Consulta: 1 septiembre 2020].

POLÍVKA, Jaroslav J. What it's like to work with Wright [ $2^{\circ}$ borrador]. s. 1.: Literatura gris, ca. 1957. J. J. Polivka papers, University Archives of the University Libraries, University at Buffalo the State University of New York. 
<https://findingaids.lib.buffalo.edu/repositories/2/archival_objects/225469>. [Consulta: 1 septiembre 2020].

POLÍVKA PAPERS, Container Inventory [en línea]. University Archives of the University Libraries, University at Buffalo the State University of New York. $<$ https://findingaids.lib.buffalo.edu/repositories/2/resources/704>. [Consulta: 1 septiembre 2020].

PONCE ORTIZ DE INSAGURBE, M. y SÁNCHEZ SÁNCHEZ, J. Construir el siglo XX con Informes de la Construcción: Índice de índices [en línea]. Madrid: Consejo Superior de Investigaciones Científicas, 2006. <https://editorial.csic.es/publicaciones/libros/11339/978-8400-08503-2/construir-el-siglo-XX-con-informes-de-la-construcc.html>. [Consulta: 1 septiembre 2020].

REGISTRO MERCANTIL DE MADRID, Notas simples y certificaciones [en línea]. <https://www.rmercantilmadrid.com/RMM/Tramites/Notas_Simples.aspx>. [Consulta: 1 septiembre 2020].

REVISTA DE OBRAS PÚBLICAS, Repositorio [en línea]. <http://ropdigital.ciccp.es/>. [Consulta: 1 septiembre 2020].

RILEM (Réunion Internationale des Laboratoires d'Essais et des Recherches sur les Matériaux et les Constructions), Web institucional [en línea]. <https://www.rilem.net/>. [Consulta: 1 septiembre 2020].

RODRÍGUEZ-LÓPEZ, Carolina y Jara MUÑOZ HERNÁNDEZ, ed. Hacia el Centenario: La Ciudad Universitaria de Madrid a sus 90 años. Madrid: Ediciones Complutense, 2018.

SÁENZ RIDRUEJO, Fernando. Una historia de la Escuela de Caminos: La Escuela de Caminos de Madrid a través de sus protagonistas. Madrid: Colegio de Ingenieros de Caminos, Canales y Puertos, Fundación Juan-Miguel Villar Mir, 2016. Ciencias, humanidades e ingeniería, 94.

SALVADORI, Mario. Torroja, pionero de la moderna arquitectura. En: Nueva forma. 1968, no. 32 , p. [57-58].

SMITH, G. E. Kidder. Soaring concrete canopies shelter the Madrid Hippodrome: Eduardo Torroja, engineer. En: Architectural Forum. 1950, vol. 92, no. 5, p. 130-131.

SPANISH ARCHITECTURE SEEN WORLDWIDE, Base de datos de referencias bibliográficas [en línea]. <https://www.mendeley.com/community/spanish-architecture-seenworldwide/>. [Consulta: 1 septiembre 2020].

TARRAGÓ, Salvador, ed. La modernidad en la obra de Eduardo Torroja. Madrid: Colegio de Ingenieros de Caminos, Canales y Puertos, Turner, 1979.

TARRAGÓ, Salvador, ed. J. Eugenio Ribera: Ingeniero de Caminos, 1864-1936. Madrid: Colegio de Ingenieros de Caminos, Canales y Puertos, 1982. 
Una cartografía de los fondos documentales del ingeniero Eduardo Toroja...

THÜRLIMANN, Bruno. Torroja visto desde el extranjero. En: E. ALARCÓN, ed., De la construcción a la ciencia: Ayer y hoy de Eduardo Torroja. Madrid: Real Academia de Ciencias, Academia de Ingeniería, 2000, p. 49-53.

TORROJA, Eduardo. Obras principales de hormigón armado proyectadas y dirigidas por Eduardo Torroja de 1926 a 1936. [Madrid]: Talleres de Unión Poligráfica, 1936.

TORROJA, Eduardo. Razón y ser de los tipos estructurales. Madrid: Artes Gráficas Mag, S.A., 1957.

TORROJA, Eduardo. Philosophy of structures. Berkeley, CA: University of California Press, 1958a.

TORROJA, Eduardo. The structures of Eduardo Torroja: An autobiography of engineering accomplishment. New York: F.W. Dodge Corporation, 1958b.

TORROJA CAVANILLAS, José Antonio. Torroja. En: Museo virtual: CEDEX. Madrid: CEDEX, 1999 [en línea]. <http://www.museovirtual.cedex.es/detalle-exposicioncedex.html?id=10> [Consulta: 1 septiembre 2020].

ZIKMUND-LENDER, Ladislav. Jaroslav J. Polívka: 1886-1960. En: E. HEYD, ed., Lístek do nového světa $=$ Ticket to the new world. Praga: Czech National Trust, 2018, p. 28-43.

Ficha bibliográfica:

GRAUS ROVIRA, Ramon. Una cartografía de los fondos documentales del ingeniero Eduardo Torroja a través de su trayectoria científica, académica y profesional. Ar@cne. Revista Electrónica de Recursos de Internet sobre Geografia y Ciencias Sociales. Barcelona: Universidad de Barcelona, I de febrero de 202I, vol. XXV, n 25I. DOI: https://doi.org/I0.I344/ara202I.25I.32295

\section{Menú Geo Crítica}

